\title{
Ultrasound-guided fine needle aspiration cytology in staging clinically node-negative invasive breast cancer
}

\author{
Daniela Huber • Cristophe Duc • Nicolas Schneider • \\ Dominique Fournier
}

Received: 7 July 2011 / Accepted: 14 October 2011 / Published online: 16 November 2011

(C) Springer-Verlag 2011

\begin{abstract}
The aim of this study was to evaluate the value of ultrasound (US)-guided axillary lymph node fine needle aspiration cytology (FNAC) in staging clinically nodenegative invasive breast cancer. Based on retrospective data, we analyzed sensitivity, specificity, and positive and negative predictive value and efficacy of preoperative axillary US-guided FNAC. A total of 108 consecutive female patients with histological-confirmed invasive breast cancer between January 2006 and December 2010 were included. The management decisions were based on cytological results. Twenty-two patients underwent neoadjuvant chemotherapy and 86 remaining patients benefited of primary surgery. Patients with positive cytology or included in neoadjuvant regimens were scheduled for axillary lymph node dissection (ALND), while patient with negative or nondiagnostic cytology underwent sentinel lymph node biopsy. Axillary US-guided FNAC was compared with definitive pathology of surgically removed lymph nodes. Axillary metastases were found in 55 out of 108 patients $(50.9 \%)$. In these cases we proceeded with ALND. Excluding the group benefiting from neoadjuvant chemotherapy, we could spare a second surgical intervention for 37 out of 86 patients $(43 \%)$. The axillary US with FNAC has a sensitivity of $73 \%$, a specificity of $85 \%$, a
\end{abstract}

D. Huber $(\bowtie) \cdot$ N. Schneider

Obstetrics and Gynecology, CHCVs Sion Hospital,

Rue Champsec 80,

Sion 1950, Switzerland

e-mail: ghetudana@gmail.com

C. Duc

Pathology, ICHV Sion Hospital,

Rue Champsec 80,

Sion 1950, Switzerland

D. Fournier

Radiology, IRS Sion Radiologic Institute,

Rue du Scex 2,

Sion 1950, Switzerland positive predictive value of $89 \%$, and a negative predictive value of $66 \%$. Without taking into account the neoadjuvant chemotherapy group, in which the statistical analyzes might be biased by the complete histological response, specificity and positive predictive value increased to $100 \%$ and negative predictive value to $71 \%$. US combined with FNAC of axillary lymph nodes is a simple, minimally invasive, and reproducible diagnostic approach in improving the preoperative axillary staging of invasive breast cancer patients.

Keywords Invasive breast cancer. Axillary node ultrasound · Axillary node cytology Sentinel lymph node

\section{Background and objective}

The current goal in oncologic breast surgery is to tailor treatment options to allow optimal care without unnecessary interventions. Breast surgery has steadily evolved from an extensive to a more conservative approach. Since 2000, many clinical trials have confirmed that sentinel lymph node biopsy (SLNB) is an accurate technique that permits omitting a complete axillary lymph node dissection (ALND) in selected patients while diminishing the incidence of arm and shoulder morbidity $[1,2]$. SLNB is developing in new directions (multifocal/multicentric tumors) [3]: applications with neoadjuvant chemotherapy [4-10], axillary reverse node mapping [11-13], and nanotechnology [14]. Axillary lymph node status is the single most significant predictive factor for patients with invasive breast tumors [15]. A positive sentinel lymph node (SLN) requires subsequent ALND. Sparing a second axillary surgery is a current concern that has stimulated the development of other approaches including molecular biology techniques for intraoperative assessment of SLN and preoperative detection of node metastases through the use of imaging methods. Ultrasound (US) is a simple and 
well-accepted method to examine axillary and non-axillary lymph nodes associated with breast cancer. Recent publications have reported that routine axillary US combined with cytology or core biopsy is an effective method to evaluate lymph node metastases prior to surgery [16-35]. Thus, pretreatment axillary US for early breast cancer patients and needle sampling of morphologically abnormal lymph nodes are now widely recommended [36]. The aim of our study was to establish the accuracy of US-guided fine needle aspiration cytology (FNAC) of axillary lymph nodes for the detection of clinically silent metastases and to find out how often a SLNB could be avoided.

\section{Material and method}

From January 2006 to December 2010, 144 consecutive patients with invasive breast cancer, clinical stage T1/2N0, were evaluated by axillary US with FNAC. Thirty-six patients were excluded due to a personal history of breast cancer, previous or ongoing chemotherapy or previous breast/axilla surgery, and non identifiable lymph node on axillary US. All 108 remaining patients underwent initial breast biopsy confirming invasive malignancy.

Both axillary US and node FNAC were performed by the same breast radiologist. A high-resolution probe $(12 \mathrm{MHz}$ electronically focused linear array transducer) of ATL HDI 500 Philips Healthcare was utilized. The suspicious ultrasound node characteristics in our study were: length/width ratio of $<1.5$, cortical asymmetrical thickness more than $3 \mathrm{~mm}$, hypoechoic cortical nodule deforming hilum, and the absence of the fatty hilum. If at least one of these criteria was observed, the node was selected for FNAC. If more than one abnormal lymph node was found, the most suspicious one was selected. If all suspect lymph nodes were similar, the lowest one in the axilla was selected. If no suspect lymph node was detected, the lowest normal lymph node larger than $5 \mathrm{~mm}$ was sampled. In one case a preoperative breast NMRI identified a suspicious internal mammary lymph node that was sampled by US-guided FNAC.

All cytological samples were processed with the Thin Prep System and analyzed by a breast specialist pathologist and classified as: insufficient for diagnosis and negative or positive for malignancy. Patients with cytology that was negative or insufficient for diagnosis were referred to SLNB. Patients with positive cytologies and all patients treated with neoadjuvant chemotherapy underwent ALND. If the intraoperative imprint cytologies (Diff-Quick staining protocol) or the extemporaneous frozen sections of SLN (hematoxylin and eosine staining method) were positive for malignancy the patients underwent immediate ALND. If the SLNB were negative based on extemporaneous examination but micro or macro metastatic on final histology (metastasis, $\geq 0.2 \mathrm{~mm}$ ) the patients underwent ALND within 2 weeks. If isolated tumor cells were identified through definitive histology, no further ALND was performed.

The total number of harvested nodes, the number of positive nodes, and the size of metastasis were recorded. The tumor size and grade, histological type, lymphovascular or perinervous infiltration, the type of surgical intervention, and the neoadjuvant treatments were also included in our data. The final pathological results of harvested sentinel lymph nodes or ALND were correlated with US-guided FNAC. The sensitivity, specificity, and positive and negative predictive values were calculated.

\section{Findings}

One hundred eight patients had axillary lymph US-guided FNAC as a part of the investigation for invasive breast cancer. No immediate or late complications such as bleeding, hematoma, nerve injury, or infection were reported. Eightysix patients underwent primary surgery and the other 22 underwent neoadjuvant chemotherapy with subsequent surgery. The median age was 58 (range 33-83) and 54 (range 3371) years for the two groups, respectively.

The most frequent histological type of primary invasive breast tumor in our group was ductal carcinoma in 82 $(76 \%)$ patients. Other described histological types were lobular carcinoma in $15(13.9 \%)$ patients, mixed carcinoma in $9(8.3 \%)$, and mucinous carcinoma in $2(1.8 \%)$ cases.

As overlap of ultrasound features between reactive nodes and suspicious/metastatic ones is documented [19, 37], our breast radiologist decided to puncture also non-suspicious nodes larger than $5 \mathrm{~mm}[19,38]$. As our study is retrospective, the ultrasound reports were nonuniform and did not mention in detail the sonographic nodes description and the reasons for choosing a suspicious or a nonsuspicious node. Hence, statistical analysis including the significance of each sonographic suspicious finding could not be made.

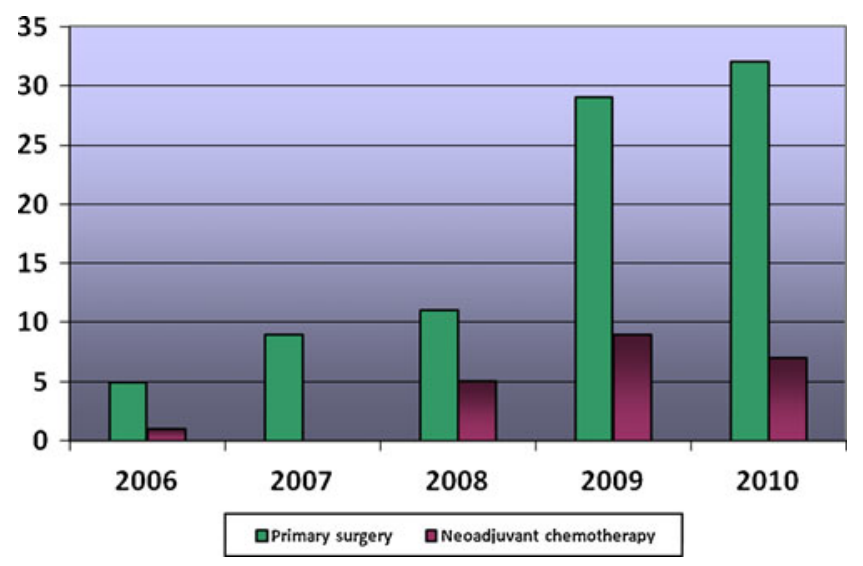

Fig. 1 Annual patient recruitment between 2006 and 2010 


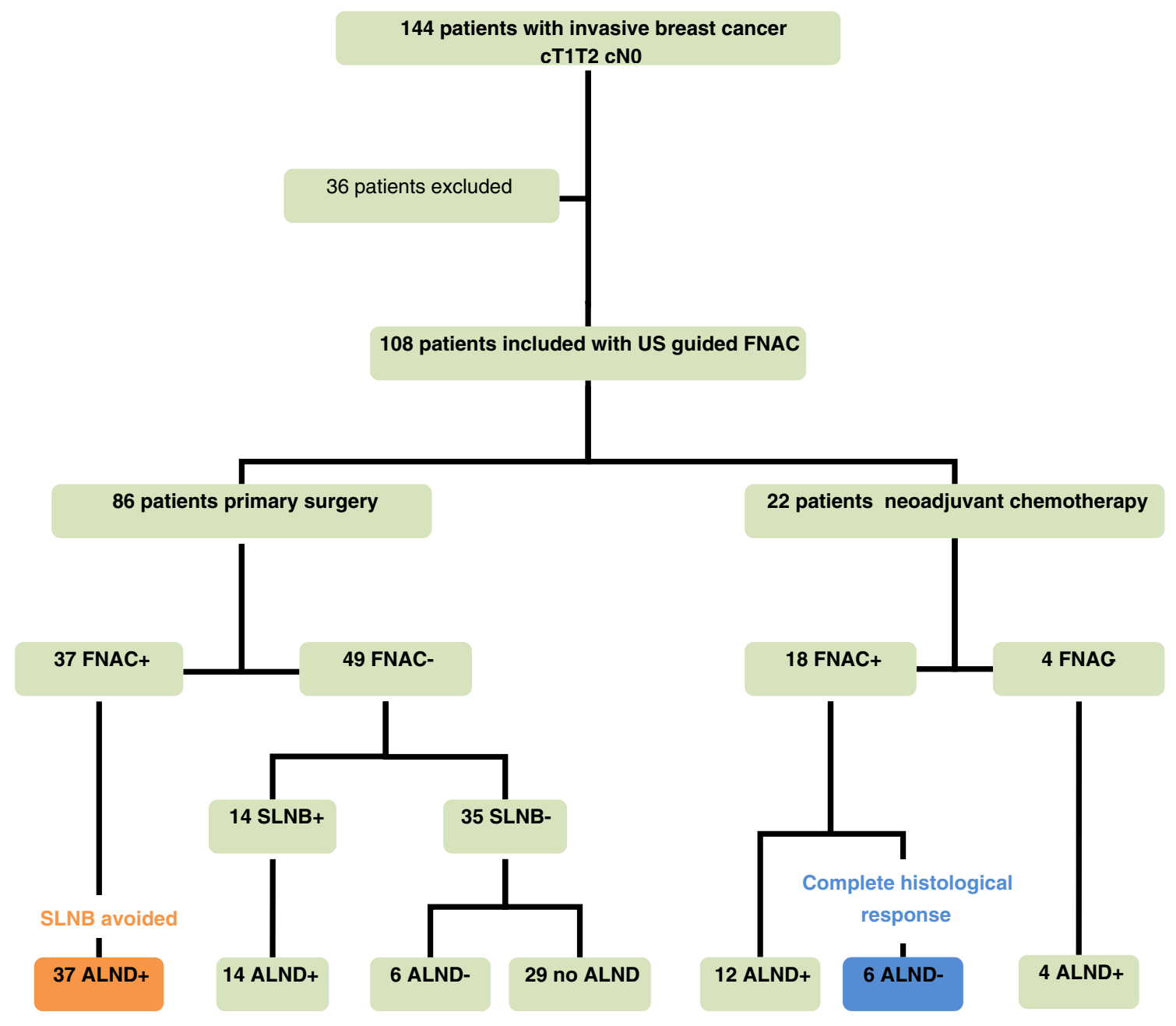

Fig. 2 Study design and results

For $55(50.9 \%)$ patients the FNAC was positive and for $46(42.6 \%)$ patients it was negative. In seven $(6.5 \%)$ cases the FNAC was insufficient for diagnosis (in one case the FNAC sampled only necrotic cells and in six other cases there was insufficient material to reach a conclusive result). All these patients were considered to be negative for statistical analysis. False-negative results were documented in $18(33.9 \%)$ out of these 53 patients (46 with negative cytology and 7 considered negative, but insufficient for diagnosis). Three patients had isolated tumor cells on

Table 1 Surgical node staging compared with preoperative FNAC (108 patients)

\begin{tabular}{lllll}
\hline & \multicolumn{2}{l}{ Surgical staging } & Total \\
\cline { 3 - 4 } & & Positive nodes & Negative nodes & \\
\hline \multirow{2}{*}{ FNAC } & Positive & 49 & 6 & 55 \\
& Negative & 18 & 35 & 53 \\
& Total & 67 & 41 & 108 \\
\hline
\end{tabular}

definitive histology despite a negative FNAC. We did not consider these results as cytohistological discordances as pathological classification remains $\mathrm{pN} 0(\mathrm{sn})$ and no further ALND was performed.

For the patients in the primary surgery group, no falsepositive result was documented. In the neoadjuvant chemotherapy group, 6 out of 18 patients with positive pretreatment FNAC had negative axillary lymph nodes. We have interpreted this apparent discrepancy as a complete node histological response and not as false-

Table 2 Surgical node staging compared with preoperative FNAC in primary surgery group

\begin{tabular}{lllll}
\hline & \multicolumn{2}{c}{ Surgical staging } & \multirow{2}{*}{ Total } \\
\cline { 3 - 4 } & & Positive nodes & Negative nodes & \\
\hline \multirow{2}{*}{ FNAC } & Positive & 37 & 0 & 37 \\
& Negative & 14 & 35 & 49 \\
& Total & 51 & 35 & 86 \\
\hline
\end{tabular}


Table 3 Lymph node morphology by axillary ultrasound and cytological findings in the "primary surgery" group

\begin{tabular}{lllll}
\hline & & \multicolumn{2}{l}{ Lymph node morphology (US) } & \multirow{2}{*}{ Total } \\
\cline { 3 - 4 } & & Normal & Suspicious & \\
\hline \multirow{2}{*}{ FNAC } & Positive & 5 & 32 & 37 \\
& Negative & 35 & 14 & 49 \\
& Total & 40 & 46 & 86 \\
\hline
\end{tabular}

positive results. Figure 1 shows the patients' recruitment from 2006 to 2010. Figure 2 describes our study design and the results. Table 1 reviews the final histological findings for the 86 patients treated by primary surgery. Table 2 compares the surgical node staging with FNAC for the primary surgery group. Table 3 summarizes the sonographic aspect of the punctured nodes and the cytological results in the same group. The data in Tables 4 and 5 review the clinicopathological features of invasive carcinomas and

Table 4 Clinicopathological features of invasive carcinoma primary surgery group

\begin{tabular}{lc}
\hline Pathological characteristics & $N(\%)$ \\
\hline Histological type & \\
Invasive ductal carcinoma: & $66 / 86(76.7 \%)$ \\
Invasive lobular carcinoma & $12 / 86(14.0 \%)$ \\
Invasive mixed carcinoma & $6 / 86(7.0 \%)$ \\
Invasive mucinous carcinoma & $2 / 86(2.3 \%)$ \\
Tumor grade & \\
G1 & $10 / 86(11.6 \%)$ \\
G2 & $57 / 86(66.3 \%)$ \\
G3 & $19 / 86(22.1 \%)$ \\
Pathological tumor stage & \\
pT1a & $2 / 86(2.2 \%)$ \\
pT1b & $5 / 86(5.8 \%)$ \\
pT1c & $31 / 86(36.0 \%)$ \\
pT2 & $39 / 86(45.3 \%)$ \\
pT3 & $8 / 86(9.6 \%)$ \\
pT4 & $1 / 86(1.1 \%)$ \\
Lymphovascular infiltration & \\
Absent & $65 / 86(75.6 \%)$ \\
Present & $21 / 86(24.4 \%)$ \\
Pathological node stage & \\
pN0 (sn) & $29 / 86$ \\
pN0 & $6 / 86$ \\
pN1(sn) & $1 / 86$ \\
pN1 & $35 / 86$ \\
pN2 & $11 / 86$ \\
Total & $4 / 86$ \\
\hline & $86(100 \%)$ \\
\hline
\end{tabular}

Table 5 Cytohistological discordances in primary surgery group

\begin{tabular}{lc}
\hline Histological findings & $n(\%)$ \\
\hline FNAC & $2(14.3 \%)$ \\
Insufficient for diagnose & $12(85.7 \%)$ \\
Negative (false negative) & $0 \%$ \\
Positive (false positive) & \\
Number of positive nodes & $11(78.5 \%)$ \\
1 lymph node & $2(14.3 \%)$ \\
2 lymph nodes & $1(7.2 \%)$ \\
3 lymph nodes & \\
Size of nodal metastasis & $6(42.8 \%)$ \\
Not documented & $3(21.4 \%)$ \\
Micrometastasis (0.2 to 2 mm) & \\
Macrometastasis & $4(28.6 \%)$ \\
2 to 4 mm & $1(7.2 \%)$ \\
$>4$ mm & \\
Pathological stage grouping & $1(7.2 \%)$ \\
IB (pT1cN1mi) & $5(35.7 \%)$ \\
IIA (pT1cN1a) & $8(57.1 \%)$ \\
IIB (pT2N1a et pT2N1b) & $14(100 \%)$ \\
Total cytohistological discordances & \\
\hline
\end{tabular}

the 14 cytohistological discordances in primary surgery group. For this group of patients, the cytohistological concordance was established in $72(83.7 \%)$ patients (37 with positive and 35 with negative results). Consequently, for 37 patients a second axillary surgery was avoided $(43.02 \%)$. Fourteen discordances were documented as either FNAC negative or insufficient for diagnosis but positive on definitive histology. In 12 patients, the FNAC was negative and in two patients the material was insufficient for cytological diagnosis. Among these 14 patients with cytohistological discordances, 11 had only one positive node, 2 patients had two positive nodes and 1 patient had three positive lymph nodes.

The internal mammary lymph node sampled by the USguided FNAC was positive for malignancy. The patient underwent ALND (14 negative axillary lymph nodes) and the final pathological stage was $\mathrm{pT} 1 \mathrm{cN} 2 \mathrm{~b}$.

\section{Discussion}

The appropriate pretreatment evaluation enables personalized management of breast cancer patients. SLNB is widely accepted today and has led to decreased shoulder and arm morbidity. Nevertheless, this technique requires the sustained coordination of a multidisciplinary team. Some patients need a second axillary surgery if the definitive SLNB with immunostaining proves the presence of node metastases. Although there are still controversies concerning morbidity differences 
between immediate and delayed ALND [39-43], operating on a distorted axilla represents a surgical challenge with emotional distress for the patient as well as considerable additional medical costs [44]. Axillary ultrasound is noninvasive, reproducible, largely available, widely accepted by patients, and cost effective [35, 44]. In addition, all lymph node chains can be evaluated (intramammary, internal mammary chain, infra- and supraclavicular, or axillary nodes).

In our study, immediate assessment of the quality of FNAC was not feasible. Seven (6.5\%) out of 108 FNAC specimens were insufficient for diagnosis. Immediate assessment of specimens by a pathologist might reduce the proportion of inadequate samples [45] and allow additional lymph node passes by the radiologist to improve quality [28]. For the 86 patients treated by primary surgery, the overall sensitivity was $72.55 \%$ and the specificity was $100 \%$ (Table 6) although identifiable non-suspicious nodes were punctured and aspirated for cytological pretreatment examination. Forty-six patients of this group had sonographic suspicious nodes. Thirty-one out of these 46 nodes were positive. By puncturing normal nodes as well, a total of 37 patients with node metastasis were identified (Table 3). The major benefit of US-guided FNAC was that it spared a second surgical intervention for these 37 patients ( $43 \%$ of the primary surgery group). For patients with neoadjuvant treatment, this approach enables the gathering of further information about the in vivo response to chemotherapy. Patients who have a complete nodal and breast pathological response enjoy a much better outcome than those who still have residual disease after therapy [46].

Recently, the NCCN guidelines (version 2.2011) introduced prechemotherapy SLNB as an option for clinically node-negative breast cancer patients benefiting from neoadjuvant regimens. As for early breast cancer patients, we expect that FNAC will allow a better

Table 6 Sensitivity, specificity, positive predictive value and negative predictive value

\begin{tabular}{|c|c|c|c|c|c|c|c|c|c|}
\hline \multirow[t]{2}{*}{ Patients } & \multirow[t]{2}{*}{$\mathrm{n}$} & \multicolumn{2}{|c|}{ FNAC+ } & \multicolumn{2}{|c|}{ FNAC- } & \multirow[t]{2}{*}{ Sensitivity } & \multirow[t]{2}{*}{ Specificity } & \multirow{2}{*}{$\begin{array}{l}\text { Positive predictive } \\
\text { value }\end{array}$} & \multirow{2}{*}{$\begin{array}{l}\text { Negative predictive } \\
\text { value }\end{array}$} \\
\hline & & $\begin{array}{l}\mathrm{N} \\
+\end{array}$ & $\begin{array}{l}\mathrm{N} \\
-\end{array}$ & $\begin{array}{l}\mathrm{N} \\
+\end{array}$ & $\begin{array}{l}\mathrm{N} \\
-\end{array}$ & & & & \\
\hline All patients & 108 & 49 & 6 & 18 & 35 & $73.13 \%$ & $85.36 \%$ & $89.09 \%$ & $66.03 \%$ \\
\hline $\begin{array}{l}\text { Neoadjuvant chemotherapy group } \\
\text { Pathological stage }\end{array}$ & 22 & 12 & 6 & 4 & 0 & $66.66 \%$ & & $75 \%$ & \\
\hline урТ0 & $2 / 22$ & 1 & 1 & 0 & 0 & & & & \\
\hline ypT1 & $6 / 22$ & 2 & 3 & 1 & 0 & & & & \\
\hline урТ2 & $8 / 22$ & 4 & 2 & 2 & 0 & & & & \\
\hline урT3/Т4 & $6 / 22$ & 5 & 0 & 1 & 0 & & & & \\
\hline Primary surgery group & 86 & 37 & 0 & 14 & 35 & $72.55 \%$ & $100 \%$ & $100 \%$ & $71.43 \%$ \\
\hline \multicolumn{10}{|l|}{ Tumor grade } \\
\hline G1 & $12 / 86$ & 3 & 0 & 1 & 8 & $75 \%$ & $100 \%$ & $100 \%$ & $88.88 \%$ \\
\hline G2 & $55 / 86$ & 26 & 0 & 11 & 18 & $70.27 \%$ & $100 \%$ & $100 \%$ & $62.07 \%$ \\
\hline $\mathrm{G} 3$ & $19 / 86$ & 8 & 0 & 3 & 8 & $72.72 \%$ & $100 \%$ & $100 \%$ & $72.72 \%$ \\
\hline \multicolumn{10}{|l|}{ Pathological stage } \\
\hline pT1 & $38 / 86$ & 11 & 0 & 6 & 21 & $64.70 \%$ & $100 \%$ & $100 \%$ & $77.77 \%$ \\
\hline pT2 & $39 / 86$ & 19 & 0 & 9 & 11 & $67.85 \%$ & $100 \%$ & $100 \%$ & $55 \%$ \\
\hline pT3-T4 & $9 / 86$ & 7 & 0 & 0 & 2 & $100 \%$ & $100 \%$ & $100 \%$ & $100 \%$ \\
\hline \multicolumn{10}{|l|}{ LVI/PNI } \\
\hline Present & $21 / 86$ & 12 & 0 & 6 & 3 & $66.66 \%$ & $100 \%$ & $100 \%$ & $30 \%$ \\
\hline Absent & $65 / 86$ & 25 & 0 & 9 & 31 & $73.53 \%$ & $100 \%$ & $100 \%$ & $77.5 \%$ \\
\hline \multicolumn{10}{|l|}{ Histological type } \\
\hline Ductal & $66 / 86$ & 26 & 0 & 15 & 25 & $63.41 \%$ & $100 \%$ & $100 \%$ & $62.5 \%$ \\
\hline Lobular & $12 / 86$ & 6 & 0 & 2 & 4 & $75 \%$ & $100 \%$ & $100 \%$ & $66.66 \%$ \\
\hline Mixed & $6 / 86$ & 5 & 0 & 1 & 0 & $83.33 \%$ & $100 \%$ & $100 \%$ & $0 \%$ \\
\hline Mucinous & $2 / 86$ & 2 & 0 & 0 & 1 & $100 \%$ & $100 \%$ & $100 \%$ & $100 \%$ \\
\hline \multicolumn{10}{|l|}{ Type of lesion } \\
\hline Unifocal & $68 / 86$ & 30 & 0 & 12 & 26 & $1.42 \%$ & $100 \%$ & $100 \%$ & $68.42 \%$ \\
\hline Multifocal/multicentric & $18 / 86$ & 7 & 0 & 3 & 8 & $70 \%$ & $100 \%$ & $100 \%$ & $72.72 \%$ \\
\hline
\end{tabular}


selection of patients for prechemotherapy SLNB and will carry the same prognostic value as SLNB if complete histological response is observed.

False-positive results have seldom been reported [29, 47]. These results have been related to misinterpreting the presence of reactive lymphoid or mesothelial cells as carcinoma infiltration. The majority of cases believed to be false-positive may, in fact, be true-positive (complete histological response or failure to detect minimal residual disease in the final histology). We believe that all positive FNAC with a negative definitive histology must be treated cautiously in order to exclude a non-harvested positive node.

False-negative FNAC results probably occurred in part due to failure to target the real SLN or the most suspected region of the lymph node and perhaps to misinterpretations including failure to recognize tumor cells. As this is a retrospective study, no information about the punctured lymph node and the correlation with the SLN surgically removed was possible.

\section{Conclusions}

With a sensitivity of $73 \%$, axillary US associated with FNAC plays an important role in preoperative lymph node staging for newly diagnosed invasive breast cancer patient. In selecting non-suspicious nodes for pretreatment lymph node cytology, we could spare a second axillary surgery for $43 \%$ of the patients. In addition, for patients included on neoadjuvant regimens, it allows a better selection for prechemotherapy SLNB and furthermore an evaluation of the histological response to treatment offering valuable information on patient prognosis and predictive factors.

Acknowledgments Many thanks go to M.E. Visher for her essential and gracious support in revising the manuscript's English.

Declaration of interest The authors report no conflicts of interest. The authors alone are responsible for the content and writing of the paper.

\section{References}

1. Veronesi U et al (2002) Twenty-year follow-up of a randomized study comparing breast conserving surgery with radical mastectomy for early breast cancer. N Engl J Med 347(16):1227-1232

2. Veronesi U et al (1997) Sentinel-node biopsy to avoid axillary dissection in breast cancer with clinically negative lymph-nodes. Lancet 349(9069): 1864-1867

3. Fearmonti RM et al (2009) False negative rate of sentinel lymph node biopsy in multicentric and multifocal breast cancers may be higher in cases with large additive tumor burden. Breast J 15 (6):645-648

4. Brady EW (2002) Sentinel lymph node mapping following neoadjuvant chemotherapy for breast cancer. Breast J 8(2):97-100
5. Classe JM et al (2009) Sentinel lymph node biopsy after neoadjuvant chemotherapy for advanced breast cancer: results of Ganglion Sentinelle et Chimiotherapie Neoadjuvante, a French prospective multicentric study. J Clin Oncol 27(5):726-732

6. Hunt KK et al (2009) Sentinel lymph node surgery after neoadjuvant chemotherapy is accurate and reduces the need for axillary dissection in breast cancer patients. Ann Surg 250 (4):558-566

7. Iwase $\mathrm{H}$ et al (2009) Advantage of sentinel lymph node biopsy before neoadjuvant chemotherapy in breast cancer treatment. Surg Today 39(5):374-380

8. Medina-Franco H, Salgado-Nesme N, Zeron-Medina-Cuairan J (2008) Sentinel lymph node biopsy after neoadjuvant systemic chemotherapy in patients with breast cancer: a prospective pilot trial. Rev Invest Clin 60(5):390-394

9. Menard JP et al (2009) Sentinel lymphadenectomy for the staging of clinical axillary node-negative breast cancer before neoadjuvant chemotherapy. Eur J Surg Oncol 35(9):916-920

10. van Deurzen $\mathrm{CH}$ et al (2009) Accuracy of sentinel node biopsy after neoadjuvant chemotherapy in breast cancer patients: a systematic review. Eur J Cancer 45(18):3124-3130

11. Casabona $\mathrm{F}$ et al (2008) Axillary reverse mapping in breast cancer: a new microsurgical lymphaticvenous procedure in the prevention of arm lymphedema. Ann Surg Oncol 15(11):3318-3319

12. Casabona $F$ et al (2009) Feasibility of axillary reverse mapping during sentinel lymph node biopsy in breast cancer patients. Ann Surg Oncol 16(9):2459-2463

13. Khan SA (2009) Axillary reverse mapping to prevent lymphedema after breast cancer surgery: defining the limits of the concept. J Clin Oncol 27(33):5494-5496

14. Johnson L, Charles-Edwards G, Douek M (2010) Nanoparticles in sentinel lymph node assessment in breast cancer. Cancers 2:18841894

15. Mainiero MB (2010) Regional lymph node staging in breast cancer: the increasing role of imaging and ultrasound-guided axillary lymph node fine needle aspiration. Radiol Clin North Am 48(5):989-997

16. Park S et al (2011) Impact of preoperative ultrasonography and fine-needle aspiration of axillary lymph nodes on surgical management of primary breast cancer. Ann Surg Oncol 18 (3): $738-744$

17. Abe $\mathrm{H}$ et al (2009) Axillary lymph nodes suspicious for breast cancer metastasis: sampling with US-guided 14-gauge core-needle biopsy-clinical experience in 100 patients. Radiology 250 (1):41-49

18. Baruah BP et al (2010) Axillary node staging by ultrasonography and fine-needle aspiration cytology in patients with breast cancer. Br J Surg 97(5):680-683

19. Bonnema J et al (1997) Ultrasound-guided aspiration biopsy for detection of nonpalpable axillary node metastases in breast cancer patients: new diagnostic method. World J Surg 21 (3):270-274

20. Britton PD et al (2009) Use of ultrasound-guided axillary node core biopsy in staging of early breast cancer. Eur Radiol 19 (3):561-569

21. Cowher MS et al (2008) Correlation of the use of axillary ultrasound and lymph node needle biopsy with surgical lymph node pathology in patients with invasive breast cancer. Am J Surg 196(5):756-759

22. Damera A et al (2003) Diagnosis of axillary nodal metastases by ultrasound-guided core biopsy in primary operable breast cancer. Br J Cancer 89(7):1310-1313

23. Davey P et al (2011) The value of axillary ultrasound with fine needle aspiration as a preoperative staging procedure in breast cancer: Northern Irish experience. Ir J Med Sci 180 (2):509-511 
24. Garcia-Ortega MJ et al (2011) Pretreatment axillary ultrasonography and core biopsy in patients with suspected breast cancer: diagnostic accuracy and impact on management. Eur J Radiol 79(1):64-72

25. Hinson JL et al (2008) The critical role of axillary ultrasound and aspiration biopsy in the management of breast cancer patients with clinically negative axilla. Ann Surg Oncol 15(1):250-255

26. Jain A et al (2008) The role of ultrasound-guided fine-needle aspiration of axillary nodes in the staging of breast cancer. Ann Surg Oncol 15(2):462-471

27. Jung $J$ et al (2010) Accuracy of preoperative ultrasound and ultrasound-guided fine needle aspiration cytology for axillary staging in breast cancer. ANZ J Surg 80(4):271-275

28. Krishnamurthy S (2009) Current applications and future prospects of fine-needle aspiration biopsy of locoregional lymph nodes in the management of breast cancer. Cancer 117(6):451-462

29. Kuenen-Boumeester V et al (2003) Ultrasound-guided fine needle aspiration cytology of axillary lymph nodes in breast cancer patients. A preoperative staging procedure. Eur J Cancer 39 (2):170-174

30. Lemos S et al (2005) Detection of axillary metastases in breast cancer patients using ultrasound and colour Doppler combined with fine needle aspiration cytology. Eur J Gynaecol Oncol 26 (2):165-166

31. MacNeill M, Arnott I, Thomas J (2011) Fine needle aspiration cytology is a valuable adjunct to axillary ultrasound in the preoperative staging of breast cancer. J Clin Pathol 64(1):42-46

32. Oruwari JU et al (2002) Axillary staging using ultrasound-guided fine needle aspiration biopsy in locally advanced breast cancer. Am J Surg 184(4):307-309

33. Ou YT et al (2008) Role of ultrasound-guided needle biopsy of ultrasonographic abnormal axillary lymph nodes in patients with breast cancer. Zhonghua Yi Xue Za Zhi 88(2):82-84

34. Swinson $C$ et al (2009) Ultrasound and fine needle aspiration cytology of the axilla in the preoperative identification of axillary nodal involvement in breast cancer. Eur J Surg Oncol 35 (11):1152-1157

35. Schiettecatte A et al (2011) Initial axillary staging of breast cancer using ultrasound-guided fine needle aspiration: a liquid-based cytology study. Cytopathology 22(1):30-35
36. Yarnold J (2009) Early and locally advanced breast cancer: diagnosis and treatment National Institute for Health and Clinical Excellence guideline 2009. Clin Oncol (R Coll Radiol) 21 (3): $159-160$

37. de Kanter AY et al (1999) Multicentre study of ultrasonographically guided axillary node biopsy in patients with breast cancer. Br J Surg 86(11):1459-1462

38. Alvarez S et al (2006) Role of sonography in the diagnosis of axillary lymph node metastases in breast cancer: a systematic review. AJR Am J Roentgenol 186(5):1342-1348

39. Husted-Madsen A et al (2008) Arm morbidity following sentinel lymph node biopsy or axillary lymph node dissection: a study from the Danish Breast Cancer Cooperative Group. Breast 17 (2):138-147

40. Husen M, Paaschburg B, Flyger HL (2006) Two-step axillary operation increases risk of arm morbidity in breast cancer patients. Breast 15(5):620-628

41. Olson JA Jr et al (2008) Impact of immediate versus delayed axillary node dissection on surgical outcomes in breast cancer patients with positive sentinel nodes: results from American College of Surgeons Oncology Group Trials Z0010 and Z0011. J Clin Oncol 26(21):3530-3535

42. Holwitt DM et al (2008) Scientific Presentation Award: the combination of axillary ultrasound and ultrasound-guided biopsy is an accurate predictor of axillary stage in clinically nodenegative breast cancer patients. Am J Surg 196(4):477-482

43. Liu CQ et al (2009) Late morbidity associated with a tumournegative sentinel lymph node biopsy in primary breast cancer patients: a systematic review. Eur J Cancer 45(9):1560-1568

44. Boughey JC et al (2010) Cost modeling of preoperative axillary ultrasound and fine-needle aspiration to guide surgery for invasive breast cancer. Ann Surg Oncol 17(4):953-958

45. Ciatto S (2004) Sentinel lymph node biopsy: sentinel node technique has drawbacks. BMJ 329(7458):170

46. Chollet P et al (2002) Prognostic significance of a complete pathological response after induction chemotherapy in operable breast cancer. Br J Cancer 86(7):1041-1046

47. Tate JJ et al (1989) Ultrasound detection of axillary lymph node metastases in breast cancer. Eur J Surg Oncol 15(2):139-141 